Journal of Universal Mathematics

Vol.4 No.2 PP.172-187 (2021)

ISSN-2618-5660

DOI: $10.33773 /$ jum. 956729

\title{
GLOBAL NONEXISTENCE OF SOLUTIONS FOR THE HIGHER ORDER KIRCHHOFF TYPE SYSTEM WITH LOGARITHMIC NONLINEARITIES
}

\author{
NAZLI IRKIL AND ERHAN PIŞKIN
}

0000-0002-9130-2893 and 0000-0001-6587-4479

\begin{abstract}
This paper deals with the system of a class of nonlinear higherorder Kirchhoff-type equations with logarithmic nonlinearities. Under the appropriate assumptions, the theorem of global nonexistence is established at positive initial energy levels.
\end{abstract}

\section{INTRODUCTION}

In this paper, we study the following initial-boundary value problem (1.1)

$$
\left\{\begin{array}{c}
u_{t t}+M\left(\left\|D^{m} u\right\|^{2}+\left\|D^{m} v\right\|^{2}\right)(-\triangle)^{m} u+(-\triangle)^{m} u_{t}=|u|^{r-2} u \ln |u|, \quad x \in \Omega, t>0, \\
v_{t t}+M\left(\left\|D^{m} u\right\|^{2}+\left\|D^{m} v\right\|^{2}\right)(-\triangle)^{m} v+(-\triangle)^{m} v_{t}=|v|^{r-2} v \ln |v|, \quad x \in \Omega, t>0, \\
u(x, 0)=u_{0}(x), \quad u_{t}(x, 0)=u_{1}(x), \quad v(x, 0)=v_{0}(x), \quad v_{t}(x, 0)=v_{1}(x), \quad x \in \Omega, \\
\frac{\partial^{i}}{\partial \nu^{2}} u(x, t)=0, \quad \frac{\partial^{i}}{\partial \nu^{2}} v(x, t)=0, i=0,1,2, \ldots m-1,
\end{array}\right.
$$

where $D u=\nabla u=\left(\frac{\partial u}{\partial x_{1}}, \frac{\partial u}{\partial x_{2}}, \ldots \frac{\partial u}{\partial x_{n}}\right)$ and $r \geq 2 \gamma+2$ are real numbers and $m \geq 1$ are positive integers. The Kirchhoff term $M(s)=\beta_{1}+\beta_{2} s^{\gamma}, \gamma>0, \beta_{1} \geq 1, \beta_{2} \geq 0$. We will take $\beta_{1}=\beta_{2}=1$ for simplify. $\Omega \subset R^{n}$ is a regular and bounded domain with smooth boundary $\partial \Omega$. And $v$ denotes the outer normal.

Problem (1.1) is a generalization of a model considered by Kirchhoff [9]. Kirchhoff type equation has in the mathematical description of small amplitude vibrations of an elastic string. In the case $M(s)=1, m=1$ and $p \geq 2$, a problem of the single wave equation of the (1.1) form becomes

$$
u_{t t}-\triangle u+f\left(u_{t}\right)=|u|^{p-2} u \ln |u| .
$$

Several results of the problem (1.2) concerning local or global existence and qualitative theory have been studied by many mathematicians(see $[1,2,4,5,6,7,10$, $13,19])$. In the case $M(s) \neq 1, m=1$ and $p \geq 2$, a problem of the single wave

Date: Received: 2021-06-23; Accepted: 2021-07-29.

2000 Mathematics Subject Classification. 35B44, 35K52.

Key words and phrases. Global nonexistence, System of higher-order, Kirchhoff type equation. 
equation of (1.1) becomes the Kirchhoff-type equation which has been investigated by many authors $[3,14,18]$.

In the case $M(s) \neq 1, m>1$ the single form of the problem (1.1) without logarithmic source terms have been discussed by many authors (see $[12,16,15,11]$ ).

Let us finally mention that wave equation system with logarithmic nonlinearies was studied by Wang et al [17]. They proved global existence and finite time blow up under the different conditions by employing the potential well method and concavity method. In [8], the authors studied (1.1) problem with nonlinear damping terms. They established global existence and decay estimates.

The rest of this work is organized as follows. In Section 3, our aim is to prove the blow up of solution for $E(0)>0$. In section 2, we give some lemmas which will be useful.

\section{Preliminaries}

Now we define the potential energy functional of problem (1.1)

$$
\begin{aligned}
J(u, v)= & \frac{1}{2}\left(\left\|D^{m} u\right\|^{2}+\left\|D^{m} v\right\|^{2}\right)+\frac{1}{2 \gamma+2}\left(\left\|D^{m} u\right\|^{2}+\left\|D^{m} v\right\|^{2}\right)^{\gamma+1} \\
& -\frac{1}{r}\left(\int_{\Omega}|u|^{r} \ln |u| d x+\int_{\Omega}|v|^{r} \ln |v| d x\right)+\frac{1}{r^{2}}\left(\|u\|_{r}^{r}+\|v\|_{r}^{r}\right)
\end{aligned}
$$

and the Nehari functional

$$
\begin{aligned}
I(u, v)= & \left(\left\|D^{m} u\right\|^{2}+\left\|D^{m} v\right\|^{2}\right)+\left(\left\|D^{m} u\right\|^{2}+\left\|D^{m} v\right\|^{2}\right)^{\gamma+1} \\
& -\left(\int_{\Omega}|u|^{r} \ln |u| d x+\int_{\Omega}|v|^{r} \ln |v| d x\right) .
\end{aligned}
$$

By (2.1) and (2.2) we obtain

$$
\begin{aligned}
J(u, v)= & \frac{I(u, v)}{r}+\frac{(r-2)}{2 r}\left(\left\|D^{m} u\right\|^{2}+\left\|D^{m} v\right\|^{2}\right) \\
& +\frac{(r-2 \gamma-2)}{2 \gamma+2}\left(\left\|D^{m} u\right\|^{2}+\left\|D^{m} v\right\|^{2}\right)^{\gamma+1} \\
& +\frac{1}{r^{2}}\left(\|u\|_{r}^{r}+\|v\|_{r}^{r}\right) .
\end{aligned}
$$

Then we can introduce the stable set

$$
W=\left\{(u, v) \in H_{0}^{m}(\Omega) \times H_{0}^{m}(\Omega): I(u, v)>0\right\} \cup\{0\},
$$

the outer space of the potential well

$$
V=\left\{(u, v) \in H_{0}^{m}(\Omega) \times H_{0}^{m}(\Omega): I(u, v)<0\right\} .
$$


We introduce the total energy

$$
\begin{aligned}
E(u, v)= & \frac{1}{2}\left(\left\|u_{t}\right\|^{2}+\left\|v_{t}\right\|^{2}\right)+\frac{1}{2}\left(\left\|D^{m} u\right\|^{2}+\left\|D^{m} v\right\|^{2}\right) \\
& +\frac{1}{2 \gamma+2}\left(\left\|D^{m} u\right\|^{2}+\left\|D^{m} v\right\|^{2}\right)^{\gamma+1} \\
& -\frac{1}{r}\left(\int_{\Omega}|u|^{r} \ln |u| d x+\int_{\Omega}|v|^{r} \ln |v| d x\right)+\frac{1}{r^{2}}\left(\|u\|_{r}^{r}+\|v\|_{r}^{r}\right) .
\end{aligned}
$$

For $(u, v) \in H_{0}^{m}(\Omega) \times H_{0}^{m}(\Omega), t \geq 0$

$$
\begin{aligned}
E(0)= & \frac{1}{2}\left(\left\|u_{1}\right\|^{2}+\left\|v_{1}\right\|^{2}\right)+\frac{1}{2}\left(\left\|D^{m} u_{0}\right\|^{2}+\left\|D^{m} v_{0}\right\|^{2}\right) \\
& +\frac{1}{2 \gamma+2}\left(\left\|D^{m} u_{0}\right\|^{2}+\left\|D^{m} v_{0}\right\|^{2}\right)^{\gamma+1} \\
& -\frac{1}{r}\left(\int_{\Omega}\left|u_{0}\right|^{r} \ln \left|u_{0}\right| d x+\int_{\Omega}\left|v_{0}\right|^{r} \ln \left|v_{0}\right| d x\right)+\frac{1}{r^{2}}\left(\left\|u_{0}\right\|_{r}^{r}+\left\|v_{0}\right\|_{r}^{r}\right) .
\end{aligned}
$$

is the initial total energy. We introduce by (2.4) and (2.3)

$$
E(u, v)=\frac{1}{2}\left(\left\|u_{t}\right\|^{2}+\left\|v_{t}\right\|^{2}\right)+J(u, v),
$$

Lemma 2.1. Let $k$ be a number with $2 \leq k<\infty$ if $n \leq 2 s$ and $2 \leq k \leq \frac{2 n}{n-2 k}$ if $n>2 s$. Then there is a constant such that

$$
\|u\|_{k} \leq C\left\|D^{m} u\right\|, \forall(u, v) \in H_{0}^{m}(\Omega) \times H_{0}^{m}(\Omega) .
$$

Lemma 2.2. $E(t)$ is a nonincreasing function for $t \geq 0$ and

$$
E^{\prime}(t)=-\left(\left\|D^{m} u_{t}\right\|^{2}+\left\|D^{m} v_{t}\right\|^{2}\right) \leq 0 .
$$

Proof. Multiplying the first equation of (1.1) by $u_{t}$ and the second equation of (1.1) by $v_{t}$, and integrating on $\Omega$, we have

$$
\begin{aligned}
& \frac{1}{2} \frac{d}{d t}\left\|u_{t}\right\|^{2}+\frac{d}{d t}\left(\frac{1}{r^{2}}\|u\|_{r}^{r}-\frac{1}{r} \int_{\Omega}|u|^{r} \ln |u| d x\right) \\
& \frac{1}{2}\left(1+\left(\left\|D^{m} u\right\|^{2}+\left\|D^{m} v\right\|^{2}\right)^{\gamma}\right) \frac{d}{d t}\left\|D^{m} u\right\|^{2} \\
= & -\int_{\Omega}\left|D^{m} u_{t}\right|^{2} d x,
\end{aligned}
$$

and

$$
\begin{aligned}
& \frac{1}{2} \frac{d}{d t}\left\|v_{t}\right\|^{2}+\frac{d}{d t}\left(\frac{1}{r^{2}}\|v\|_{r}^{r}-\frac{1}{r} \int_{\Omega}|v|^{r} \ln |v| d x\right) \\
& \frac{1}{2}\left(1+\left(\left\|D^{m} u\right\|^{2}+\left\|D^{m} v\right\|^{2}\right)^{\gamma}\right) \frac{d}{d t}\left\|D^{m} v\right\|^{2} \\
= & -\int_{\Omega}\left|D^{m} v_{t}\right|^{2} d x .
\end{aligned}
$$


A summarization of (2.8) and (2.9) hence gives

$$
\begin{aligned}
& \frac{1}{2} \frac{d}{d t}\left(\left\|u_{t}\right\|^{2}+\left\|v_{t}\right\|^{2}\right) \\
+ & \frac{1}{2}\left(1+\left(\left\|D^{m} u\right\|^{2}+\left\|D^{m} v\right\|^{2}\right)^{\gamma}\right) \frac{d}{d t}\left(\left\|D^{m} u\right\|^{2}+\left\|D^{m} v\right\|^{2}\right) \\
& \frac{d}{d t}\left(-\frac{1}{r}\left(\int_{\Omega}|u|^{r} \ln |u| d x+\int_{\Omega}|v|^{r} \ln |v| d x\right)+\frac{1}{r^{2}}\left(\|u\|_{r}^{r}+\|v\|_{r}^{r}\right)\right) \\
(2.10)= & -\left(\int_{\Omega}\left|D^{m} u_{t}\right|^{2} d x+\int_{\Omega}\left|D^{m} v_{t}\right|^{2} d x\right) .
\end{aligned}
$$

Integrating (2.10) with respect to $t$ on $[0, t]$, we arrive at

$$
\begin{aligned}
& \frac{1}{2}\left(\left\|u_{t}\right\|^{2}+\left\|v_{t}\right\|^{2}\right)+\frac{1}{2}\left(\left\|D^{m} u\right\|^{2}+\left\|D^{m} v\right\|^{2}\right) \\
+ & \frac{1}{2 \gamma+2}\left(\left\|D^{m} u\right\|^{2}+\left\|D^{m} v\right\|^{2}\right)^{\gamma+1}+\frac{1}{r^{2}}\left(\|u\|_{r}^{r}+\|v\|_{r}^{r}\right) \\
& -\frac{1}{r}\left(\int_{\Omega}|u|^{r} \ln |u| d x+\int_{\Omega}|v|^{r} \ln |v| d x\right) \\
+ & \frac{1}{2}\left(\left\|u_{0}^{t}\right\| D^{2}+\left\|v_{\tau}\right\|^{2}\right)+\frac{1}{2}\left(\left\|D^{m} u_{0}\right\|^{2}+\left\|D^{m} v_{0}\right\|^{2}\right) \\
+ & \frac{1}{2 \gamma+2}\left(\left\|D^{m} u_{0}\right\|^{2}+\left\|D^{m} v_{0}\right\|^{2}\right)^{\gamma+1}+\frac{1}{r^{2}}\left(\left\|u_{0}\right\|_{r}^{r}+\left\|v_{0}\right\|_{r}^{r}\right) \\
& -\frac{1}{r}\left(\int_{\Omega}\left|u_{0}\right|^{r} \ln \left|u_{0}\right| d x+\int_{\Omega}\left|v_{0}\right|^{r} \ln \left|v_{0}\right| d x\right) .
\end{aligned}
$$

By using the definition of total energy and initial total energy, we restate (2.11) as

$$
E(t)+\left(\int_{0}^{t}\left\|D^{m} u_{\tau}\right\|^{2} d \tau+\int_{\Omega}\left\|D^{m} v_{\tau}\right\|^{2} d \tau\right)=E(0) .
$$

Now, we give some properties related with $J(u, v)$ and $I(u, v)$, respectively.

Lemma 2.3. For any $(u, v) \in H_{0}^{m}(\Omega) \times H_{0}^{m}(\Omega),\left\|D^{m} u\right\| \neq 0$ and $\left\|D^{m} u\right\| \neq 0$, let $g(\lambda)=J(\lambda u, \lambda v)$. Then we have

i) $\lim _{\lambda \rightarrow 0} g(\lambda)=0, \lim _{\lambda \rightarrow \infty} g(\lambda)=-\infty$,

ii) There is a unique $\lambda^{*}$ such that $g^{\prime}(\lambda)=0$, 
iii) Then we have

$$
I(\lambda u, \lambda v)=\lambda g^{\prime}(\lambda)\left\{\begin{array}{cc}
>0, & 0 \leq \lambda<\lambda^{*} \\
=0, & \lambda=\lambda^{*} \\
<0, & \lambda^{*}<\lambda .
\end{array}\right.
$$

Proof. By the definition of $J(u, v)$, we obtain

$$
\begin{aligned}
g(\lambda)= & J(\lambda u, \lambda v) \\
= & \frac{1}{2} \lambda^{2}\left(\left\|D^{m} u\right\|^{2}+\left\|D^{m} v\right\|^{2}\right)+\frac{1}{r^{2}} \lambda^{r}\left(\|u\|_{r}^{r}+\|v\|_{r}^{r}\right) \\
& -\frac{1}{r} \ln |\lambda| \lambda^{r}\left(\|u\|_{r}^{r}+\|v\|_{r}^{r}\right)-\frac{1}{r} \lambda^{r}\left(\int_{\Omega} u^{r} \ln |u| d x+\int_{\Omega} v^{r} \ln |v| d x\right) \\
& +\frac{1}{2 \gamma+2} \lambda^{2 \gamma+2}\left(\left\|D^{m} u\right\|^{2}+\left\|D^{m} v\right\|^{2}\right)^{2 \gamma+2} .
\end{aligned}
$$

Since $\left\|D^{m} u\right\| \neq 0$, and $\left\|D^{m} v\right\| \neq 0, \lim _{\lambda \rightarrow 0} g(\lambda)=0, \lim _{\lambda \rightarrow \infty} g(\lambda)=-\infty$. Now, differentiating $g(\lambda)$ with respect to $\lambda$, we have

$$
\begin{aligned}
g^{\prime}(\lambda)= & \lambda\left(\left\|D^{m} u\right\|^{2}+\left\|D^{m} v\right\|^{2}\right)+\lambda^{2 \gamma+1}\left(\left\|D^{m} u\right\|^{2}+\left\|D^{m} v\right\|^{2}\right)^{2 \gamma+2} \\
& -\lambda^{r-1}\left(\int_{\Omega} u^{r} \ln |u| d x+\int_{\Omega} v^{r} \ln |v| d x\right)-\lambda^{r-1} \ln |\lambda|\left(\|u\|_{r}^{r}+\|v\|_{r}^{r}\right) \\
= & \lambda\left(\left(\left\|D^{m} u\right\|^{2}+\left\|D^{m} v\right\|^{2}\right)+\beta_{2} \lambda^{2 \gamma}\left(\left\|D^{m} u\right\|^{2}+\left\|D^{m} v\right\|^{2}\right)^{2 \gamma+2}\right. \\
(2.14) \quad & \left.-\lambda^{r-2}\left(\int_{\Omega} u^{r} \ln |u| d x+\int_{\Omega} v^{r} \ln |v| d x\right)-\lambda^{r-2} \ln |\lambda|\left(\|u\|_{r}^{r}+\|v\|_{r}^{r}\right)\right) .
\end{aligned}
$$

$$
\begin{aligned}
\psi(\lambda)= & \lambda^{2 \gamma}\left(\left\|D^{m} u\right\|^{2}+\left\|D^{m} v\right\|^{2}\right)^{2 \gamma+2} \\
& -\lambda^{r-2}\left(\int_{\Omega} u^{r} \ln |u| d x+\int_{\Omega} v^{r} \ln |v| d x\right) \\
& -\lambda^{r-2} \ln |\lambda|\left(\|u\|_{r}^{r}+\|v\|_{r}^{r}\right) .
\end{aligned}
$$

Then from $2 \gamma \leq r-2$ we can deduce that $\lim _{\lambda \rightarrow \infty} \psi(\lambda)=-\infty, \psi(\lambda)$ is monotone decreasing when $\lambda>\lambda^{1}$ and there exists a unique $\lambda^{1}$ such that $\psi\left(\lambda^{1}\right)=0$. Then we obtain there is a $\lambda^{*}>\lambda^{1}$ such that $\lambda\left[\left(\left\|D^{m} u\right\|^{2}+\left\|D^{m} v\right\|^{2}\right)+\psi(\lambda)\right]=0$, which means $g^{\prime}(\lambda)=0$.

The last property (iii), is only a simple corollary of the fact that

$$
\lambda \frac{d J(\lambda u, \lambda v)}{d \lambda}=\lambda g^{\prime}(\lambda)=I(\lambda u, \lambda v) .
$$


Lemma 2.4. i) The definition of the potential well depth

$$
d=\inf _{u \in N} J(u, v),
$$

where

$$
N=\left\{(u, v):(u, v) \in H_{0}^{m}(\Omega) \times H_{0}^{m}(\Omega) \backslash\{0\}: I(u, v)=0\right\},
$$

is equivalent to

$$
d=\inf \left\{\sup _{\lambda \geq 0} J(\lambda u, \lambda v) \mid(u, v) \in H_{0}^{m}(\Omega) \times H_{0}^{m}(\Omega),\left\|D^{m} u\right\|^{2} \neq 0,\left\|D^{m} v\right\|^{2} \neq 0\right\} .
$$

ii) The constant $d$ in (2.16) satisfies

$$
d=\frac{(r-2)}{2 r}\left(\frac{1}{C_{1}^{r+1}}\right)^{\frac{2}{r-1}}
$$

where $C_{1}$ is the optimal constant of Lemma $2.1\left(H_{0}^{m}(\Omega) \hookrightarrow L^{r+1}\right)$ and

$$
\left\{\begin{array}{cc}
2 \gamma+2 \leq r \leq \frac{n+2 m}{n-2 m}, & n>2 m \\
2 \gamma+2 \leq r \leq \infty, & n \leq 2 m
\end{array}\right.
$$

Proof. i) The definition of $d$ from (iii) of Lemma 2.3 it implies that for any $(u, v) \in$ $H_{0}^{m}(\Omega) \times H_{0}^{m}(\Omega)$, there exist a $\lambda^{*}$ such that $I\left(\lambda^{*} u, \lambda^{*} v\right)=0$, that is $\left(\lambda^{*} u, \lambda^{*} v\right) \in$ $N$. By the definition of $d$ we obtain

$$
J\left(\lambda^{*} u, \lambda^{*} v\right) \geq d \text { for any }(u, v) \in H_{0}^{m}(\Omega) \times H_{0}^{m}(\Omega) /\{0\} .
$$

And because of Lemma 2.3

$$
\sup _{\lambda \geq 0} J(\lambda u, \lambda v)=J\left(\lambda^{*} u, \lambda^{*} v\right),
$$

which by virtue of (2.19) means

$$
\inf _{(u, v) \in H_{0}^{m}(\Omega) \times H_{0}^{m}(\Omega)} \sup _{\lambda \geq 0} J(\lambda u, \lambda v)=\inf _{(u, v) \in H_{0}^{m}(\Omega) \times H_{0}^{m}(\Omega)} J\left(\lambda^{*} u, \lambda^{*} v\right) \geq d,
$$

As $(u, v) \in H_{0}^{m}(\Omega) \times H_{0}^{m}(\Omega) /\{0\}$, we obtain $d$ is not equivalent to 0 , which gives (2.17). On the other hand, from the definition of $d$ given by (2.17) it implies that there exists $\lambda^{1}$ such that

$$
\sup _{\lambda \geq 0} J(\lambda u, \lambda v)=\sup J\left(\lambda^{1} u, \lambda^{1 *} v\right) .
$$

Then from Lemma 2.3 we can deduce $\lambda^{*}=\lambda^{1}$. And it shows that

$$
I\left(\lambda^{1} u, \lambda^{1} v\right)=I\left(\lambda^{*} u, \lambda^{*} v\right)=0,
$$

which means $\left(\lambda^{1} u, \lambda^{1} v\right) \in N$. By the definition of $d$, we get,

$$
d=\inf _{\left(\lambda^{*} u, \lambda^{*} v\right) \in N} J\left(\lambda^{1} u, \lambda^{1} v\right),
$$

that is

$$
d=\inf _{(u, v) \in N} J(u, v) .
$$

This complete our proof for (i). 
ii) By virtue of $I(u, v)=0$ and definition of $I(u, v)$ and the embedding theorems we obtain

$$
\begin{aligned}
\left(\left\|D^{m} u\right\|^{2}+\left\|D^{m} v\right\|^{2}\right)+ & \left(\left\|D^{m} u\right\|^{2}+\left\|D^{m} v\right\|^{2}\right)^{\gamma+1}=\int_{\Omega}|u|^{r} \ln |u| d x+\int_{\Omega}|v|^{r} \ln |v| d x, \\
\left(\left\|D^{m} u\right\|^{2}+\left\|D^{m} v\right\|^{2}\right) & \leq \int_{\Omega}|u|^{r} \ln |u| d x+\int_{\Omega}|v|^{r} \ln |v| d x \\
& \leq\|u\|_{r+1}^{r+1}+\|v\|_{r+1}^{r+1} \\
& \leq C_{1}^{r+1}\left(\left\|D^{m} u\right\|^{r+1}+\left\|D^{m} v\right\|^{r+1}\right) \\
& \leq C_{1}^{r+1}\left(\left\|D^{m} u\right\|^{2}+\left\|D^{m} v\right\|^{2}\right)^{\frac{r-1}{2}}\left(\left\|D^{m} u\right\|^{2}+\left\|D^{m} v\right\|^{2}\right),
\end{aligned}
$$

which means

$$
\left\|D^{m} u\right\|^{2}+\left\|D^{m} v\right\|^{2} \geq\left(\frac{1}{C_{1}^{r+1}}\right)^{\frac{2}{r-1}} .
$$

From the definition of $d$, we have $(u, v) \in N$. By the definition of $J(u, v),(2.22)$, (2.3) and $I(u, v)=0$, we get

$$
\begin{aligned}
J(u, v)= & \frac{I(u, v)}{r}+\frac{(r-2)}{2 r}\left(\left\|D^{m} u\right\|^{2}+\left\|D^{m} v\right\|^{2}\right) \\
& +\frac{(r-2 \gamma-2)}{2 \gamma+2}\left(\left\|D^{m} u\right\|^{2}+\left\|D^{m} v\right\|^{2}\right)^{\gamma+1}+\frac{1}{r^{2}}\left(\|u\|_{r}^{r}+\|v\|_{r}^{r}\right) \\
\geq & \frac{(r-2)}{2 r}\left(\left\|D^{m} u\right\|^{2}+\left\|D^{m} v\right\|^{2}\right) \\
\geq & \frac{(r-2)}{2 r}\left(\frac{1}{C_{1}^{r+1}}\right)^{\frac{2}{r-1}}
\end{aligned}
$$

where $2 \gamma \leq r-2$. Combining of (2.21) and (2.23), we can see clearly that

$$
d=\frac{(r-2)}{2 r}\left(\frac{1}{C_{1}^{r+1}}\right)^{\frac{2}{r-1}} .
$$

Lemma 2.5. Let $(u, v)$ be a weak solution problem of (1.1) and $\left(u_{0}, v_{0}\right) \in H_{0}^{r_{1}}(\Omega) \times$ $H_{0}^{r_{2}}(\Omega),\left(u_{1}, v_{1}\right) \in L^{2}(\Omega) \times L^{2}(\Omega)$. Suppose that $E(0)<d$

i) if $\left(u_{0}, v_{0}\right) \in W$, then $(u, v) \in W$ for $0 \leq t \leq T$

ii) if $\left(u_{0}, v_{0}\right) \in V$, then $(u, v) \in V$ for $0 \leq t \leq T$,

where $T$ is the maximum existence time of $(u(t), v(t))$.

Proof. We only prove case (i), case (ii) is similar. Let $(u(t), v(t))$ be a weak solution problem of (1.1) under the conditions and $\left(u_{0}, v_{0}\right) \in W$ and $T$ can define of the maximum existence time of $(u(x, t), v(x, t))$. Then by $(2.7)$ the energy functional is nonincreasing about $t$. So that, we have $E((u(t), v(t)))<E(0)<d$ which means $I((u(t), v(t)))>0$ for $0<t<T$. We will use contradiction and we suppose that; there is a $t_{1} \in(0, T)$ such that $I\left(u\left(t_{1}\right), v\left(t_{1}\right)\right)<0$. In this way there is 
a $t^{*} \in(0, T)$ to make $I\left(u\left(t^{*}\right), v\left(t^{*}\right)\right)=0$ because of continuity of $I(u(t), v(t))$ about time. Then by (2.16), we get

$$
d>E(0) \geq E\left(u\left(t^{*}\right), v\left(t^{*}\right)\right) \geq J\left(u\left(t^{*}\right), v\left(t^{*}\right)\right) \geq d,
$$

which is a contradiction.

Lemma 2.6. Under the condition of Lemma 2.5 (ii), we get

$$
d<\frac{(r-2)}{2 r}\left(\left\|D^{m} u\right\|^{2}+\left\|D^{m} v\right\|^{2}\right) .
$$

Proof. By using definition of the $d$, we get

$$
d=\frac{(r-2)}{2 r}\left(\frac{1}{C_{1}^{r+1}}\right)^{\frac{2}{r-1}},
$$

which together $I(u, v)<0$. Then similar calculations at $(2.22)$, we get

$$
\left\|D^{m} u\right\|^{2}+\left\|D^{m} v\right\|^{2} \geq\left(\frac{1}{C_{1}^{r+1}}\right)^{\frac{2}{r-1}}
$$

which means

$$
d<\frac{(r-2)}{2 r}\left(\frac{1}{C_{1}^{r+1}}\right)^{\frac{2}{r-1}} .
$$

\section{Finite time Blow up of SOlutions FOR POSITIVE INITIAL ENERGY}

In tis part we introduce the finite time blow up solution to problem (1.1) with $E(0)>0$. Now we give some lemmas which will be used the proof of the Theorem 3.3 .

Lemma 3.1. Let $(u, v)$ be a weak solution problem of (1.1) and $\left(u_{0}, v_{0}\right) \in H_{0}^{m}(\Omega) \times$ $H_{0}^{m}(\Omega),\left(u_{1}, v_{1}\right) \in H_{0}^{m}(\Omega) \times H_{0}^{m}(\Omega)$. Suppose that $E(0)>0$ and initial data supplies

$$
\left\|D^{m} u_{0}\right\|^{2}+\left\|D^{m} v_{0}\right\|^{2}+2\left(u_{0}, u_{1}\right)+2\left(v_{0}, v_{1}\right)>\frac{2 r(C+2)}{(r-2) C} E(0)>0,
$$

where $C$ is the best constant of Lemma 2.1.

By $(u, v) \in V$, the map

$$
\left\{t \mapsto\left\|D^{m} u\right\|^{2}+\left\|D^{m} v\right\|^{2}+2\left(u, u_{t}\right)+2\left(v, v_{t}\right)\right\}
$$

is strictly increasing.

Proof. Defining the following auxiliary function

$$
G(t)=\left\|D^{m} u\right\|^{2}+\left\|D^{m} v\right\|^{2}+2\left(u, u_{t}\right)+2\left(v, v_{t}\right),
$$

where

$$
G(0)=\left\|D^{m} u_{0}\right\|^{2}+\left\|D^{m} v_{0}\right\|^{2}+2\left(u_{0}, u_{1}\right)+2\left(v_{0}, v_{1}\right) .
$$


By taking derivative of above function, we get

$$
\begin{aligned}
G^{\prime}(t)= & 2\left(D^{m} u, D^{m} u_{t}\right)+2\left(D^{m} v, D^{m} v_{t}\right) \\
& +2\left(\left\|u_{t}\right\|^{2}+\left\|v_{t}\right\|^{2}\right)+2\left[\left(u, u_{t t}\right)+\left(v, v_{t t}\right)\right] \\
= & 2\left(\left\|u_{t}\right\|^{2}+\left\|v_{t}\right\|^{2}\right)-2 I(u, v) .
\end{aligned}
$$

By $I(u, v)<0$, for all $t \in[0, \infty)$ it gives that

$$
G^{\prime}(t)>0 .
$$

From (3.1), (3.3) and (3.4) we obtain

$$
G(t)>G(0)>0,
$$

which gives that the map

$$
\left\{t \mapsto\left\|D^{m} u\right\|^{2}+\left\|D^{m} v\right\|^{2}+2\left(u, u_{t}\right)+2\left(v, v_{t}\right)\right\}
$$

is strictly increasing.

Lemma 3.2. Under the conditions of Lemma $3.1(u, v)$ is the solution of problem (1.1) with the maximum existence time interval $[0, T)$ and $T \leq \infty$. If $\left(u_{0}, v_{0}\right) \in V$, then the all solutions $(u, v)$ belong to $V$.

Proof. Our purpose is to show that $(u, v) \in V$. Arguing by contradiction, we consider that $t^{*} \in(0, T)$ is the first time which satisfies

$$
I\left(u\left(t^{*}\right), v\left(t^{*}\right)\right)=0,
$$

and

$$
I(u(t), v(t))<0 \text { for } t \in\left[0, t^{*}\right) .
$$

Then from Lemma 3.1 and the continuity of $(u, v)$ and $\left(u_{t}, v_{t}\right)$ in $t$, for $t \in\left(0, t^{*}\right)$ we get

$$
\begin{aligned}
& \left\|D^{m} u\right\|^{2}+\left\|D^{m} v\right\|^{2}+2\left(u, u_{t}\right)+2\left(v, v_{t}\right) \\
> & \left\|D^{m} u_{0}\right\|^{2}+\left\|D^{m} v_{0}\right\|^{2}+2\left(u_{0}, u_{1}\right)+2\left(v_{0}, v_{1}\right) \\
> & \frac{2 r(C+2)}{(r-2) C} E(0) .
\end{aligned}
$$

By (2.3), (2.6) and (2.12) we arrive at

$$
\begin{aligned}
E(0)= & E(t)+\left(\int_{0}^{t}\left\|D^{m} u_{\tau}\right\|^{2} d \tau+\int_{\Omega}\left\|D^{m} v_{\tau}\right\|^{2} d \tau\right) \\
= & \frac{1}{2}\left(\left\|u_{t}\right\|^{2}+\left\|v_{t}\right\|^{2}\right)+\frac{I(t)}{r}+\frac{(r-2)}{2 r}\left(\left\|D^{m} u\right\|^{2}+\left\|D^{m} v\right\|^{2}\right) \\
& +\frac{(r-2 \gamma-2)}{2 \gamma+2}\left(\left\|D^{m} u\right\|^{2}+\left\|D^{m} v\right\|^{2}\right)^{\gamma+1}+\frac{1}{r^{2}}\left(\|u\|_{r}^{r}+\|v\|_{r}^{r}\right) \\
& +\left(\int_{0}^{t}\left\|D^{m} u_{\tau}\right\|^{2} d \tau+\int_{\Omega}\left\|D^{m} v_{\tau}\right\|^{2} d \tau\right) \\
\geq & \frac{1}{2}\left(\left\|u_{t}\right\|^{2}+\left\|v_{t}\right\|^{2}\right)+\frac{I(t)}{r}+\frac{(r-2)}{2 r}\left(\left\|D^{m} u\right\|^{2}+\left\|D^{m} v\right\|^{2}\right) .
\end{aligned}
$$


By using $r \geq 2 \gamma+2, I\left(u\left(t^{*}\right), v\left(t^{*}\right)\right)=0$, Young's inequality and Lemma 211, we conclude that

$$
\begin{aligned}
E(0) \geq & E\left(t^{*}\right) \\
\geq & \frac{1}{2}\left(\left\|u_{t}\left(t^{*}\right)\right\|^{2}+\left\|v_{t}\left(t^{*}\right)\right\|^{2}\right)+\frac{I\left(t^{*}\right)}{r}+\frac{(r-2)}{2 r}\left(\left\|D^{m} u\left(t^{*}\right)\right\|^{2}+\left\|D^{m} v\left(t^{*}\right)\right\|^{2}\right) \\
\geq & \left(\frac{1}{2}-\frac{1}{r}\right)\left(\left\|u_{t}\left(t^{*}\right)\right\|^{2}+\left\|v_{t}\left(t^{*}\right)\right\|^{2}\right)+\frac{(r-2)}{2 r}\left(\left\|D^{m} u\left(t^{*}\right)\right\|^{2}+\left\|D^{m} v\left(t^{*}\right)\right\|^{2}\right) \\
\geq & \frac{(r-2) C}{2 r(C+2)}\left(\left\|u_{t}\left(t^{*}\right)\right\|^{2}+\left\|v_{t}\left(t^{*}\right)\right\|^{2}\right)+\frac{(r-2)}{2 r}\left(\left\|D^{m} u\left(t^{*}\right)\right\|^{2}+\left\|D^{m} v\left(t^{*}\right)\right\|^{2}\right) \\
= & \frac{(r-2) C}{2 r(C+2)}\left(\left\|u_{t}\left(t^{*}\right)\right\|^{2}+\left\|v_{t}\left(t^{*}\right)\right\|^{2}+\left\|D^{m} u\left(t^{*}\right)\right\|^{2}+\left\|D^{m} v\left(t^{*}\right)\right\|^{2}\right) \\
& +\frac{(r-2)}{r(C+2)}\left(\left\|D^{m} u\left(t^{*}\right)\right\|^{2}+\left\|D^{m} v\left(t^{*}\right)\right\|^{2}\right) \\
\geq & \frac{(r-2) C}{2 r(C+2)}\left(\left\|u_{t}\left(t^{*}\right)\right\|^{2}+\left\|v_{t}\left(t^{*}\right)\right\|^{2}+\left\|D^{m} u\left(t^{*}\right)\right\|^{2}+\left\|D^{m} v\left(t^{*}\right)\right\|^{2}\right) \\
& +\frac{(r-2) C}{r(C+2)}\left(\left\|u\left(t^{*}\right)\right\|^{2}+\left\|v\left(t^{*}\right)\right\|^{2}\right) \\
\geq & \frac{(r-2) C}{2 r(C+2)}\left[\left\|u_{t}\left(t^{*}\right)\right\|^{2}+\left\|v_{t}\left(t^{*}\right)\right\|^{2}\right. \\
& \left.+\left\|D^{m} u\left(t^{*}\right)\right\|^{2}+\left\|D^{m} v\left(t^{*}\right)\right\|^{2}+\left(\left\|u\left(t^{*}\right)\right\|^{2}+\left\|v\left(t^{*}\right)\right\|^{2}\right)\right] \\
\geq & \frac{(r-2) C}{2 r(C+2)}\left\{\left[2\left(u_{t}\left(t^{*}\right), u\left(t^{*}\right)\right)+2\left(v_{t}\left(t^{*}\right), v\left(t^{*}\right)\right)\right]\right. \\
& \left.+\left\|D^{m} u\left(t^{*}\right)\right\|^{2}+\left\|D^{m} v\left(t^{*}\right)\right\|^{2}\right\} .
\end{aligned}
$$

Clearly, we show that (3.7) contradicts (3.5). This completes the proof of lemma.

Theorem 3.3. Let $(u, v)$ be a weak solution of problem of (1.1) and $\left(u_{0}, v_{0}\right) \in$ $H_{0}^{m}(\Omega) \times H_{0}^{m}(\Omega),\left(u_{1}, v_{1}\right) \in H_{0}^{m}(\Omega) \times H_{0}^{m}(\Omega)$. Suppose that (3.1) holds. Therefore the solution of problem (1.1) blows up in finite time as long as $E(0)>0$ and $\left(u_{0}, v_{0}\right) \in V$.

Proof. We prove the finite time blow up of solution to (1.1). If it is not this case, we suppose existence time $T=\infty$. For any $T_{0}>0$, we define the auxiliary function

$$
\begin{aligned}
\Phi(t)= & \|u\|^{2}+\|v\|^{2}+\int_{0}^{t}\left(\left\|D^{m} u\right\|^{2}+\left\|D^{m} v\right\|^{2}\right) d \tau \\
& \left(T_{0}-t\right)\left(\left\|D^{m} u\right\|^{2}+\left\|D^{m} v\right\|^{2}\right) .
\end{aligned}
$$

It is clear that $\Phi(t)>0$ for all $t \in\left[0, T_{0}\right]$. In view of continuity of $\Phi(t)$ in $t$, we obtain that there is a $\xi>0$ which is independent on $T_{0}$ such that

$$
\Phi(t)>\xi
$$


Then by $t \in\left[0, T_{0}\right]$, we derive

$$
\begin{aligned}
\Phi^{\prime}(t)= & 2\left(\int_{\Omega} u u_{t} d x+\int_{\Omega} v v_{t} d x\right) \\
& +\left(\left\|D^{m} u\right\|^{2}+\left\|D^{m} v\right\|^{2}\right)-\left(\left\|D^{m} u_{0}\right\|^{2}+\left\|D^{m} v_{0}\right\|^{2}\right) \\
= & 2\left(\int_{\Omega} u u_{t} d x+\int_{\Omega} v v_{t} d x\right) \\
& +2\left(\int_{0}^{t}\left(D^{m} u(\tau), D^{m} u_{\tau}(\tau)\right)+\left(D^{m} v(\tau), D^{m} v_{\tau}(\tau)\right)\right)
\end{aligned}
$$

and

$$
\begin{aligned}
\Phi^{\prime \prime}(t)= & 2\left(\left\|u_{t}\right\|^{2}+\left\|v_{t}\right\|^{2}\right)+2\left(u, u_{t t}\right)+2\left(v, v_{t t}\right) \\
& 2\left(D^{m} u, D^{m} u_{t}\right)+2\left(D^{m} v, D^{m} v_{t}\right) \\
= & 2\left(\left\|u_{t}\right\|^{2}+\left\|v_{t}\right\|^{2}\right)-2 I(u, v) .
\end{aligned}
$$

From (3.10) it implies

$$
\begin{aligned}
\left(B^{\prime}(t)\right)^{2}= & 4\left(\left(u, u_{t}\right)^{2}+\left(v, v_{t}\right)^{2}\right) \\
& +4\left(\int_{0}^{t}\left(D^{m} u(\tau), D^{m} u_{\tau}(\tau)\right)+\left(D^{m} v(\tau), D^{m} v_{\tau}(\tau)\right)\right)^{2} \\
& \left.+8\left(\int_{0}^{t}\left(D^{m} u(\tau), D^{m} u_{\tau}(\tau)\right)+\left(D^{m} v(\tau), D^{m} v_{\tau}(\tau)\right) d \tau\right)\right) .
\end{aligned}
$$

Our aim is to estimate each terms in (3.12) by Cauchy-Schwarz and Young's inequalities. We obtain the first and second terms as follow

$$
\begin{aligned}
\left(u, u_{t}\right)^{2}+\left(v, v_{t}\right)^{2} & \leq\left(\|u\|\left\|u_{t}\right\|+\|v\|\left\|v_{t}\right\|\right)^{2} \\
& \leq\left(\|u\|^{2}+\|v\|^{2}\right)\left(\left\|u_{t}\right\|^{2}+\left\|v_{t}\right\|^{2}\right)
\end{aligned}
$$


and

$$
\begin{aligned}
& \left(\int_{0}^{t}\left(D^{m} u(\tau), D^{m} u_{\tau}(\tau)\right)+\left(D^{m} v(\tau), D^{m} v_{\tau}(\tau)\right) d \tau\right)^{2} \\
\leq & \left(\int_{0}^{t}\left\|D^{m} u(\tau)\right\|\left\|D^{m} u_{\tau}(\tau)\right\|+\left\|D^{m} v(\tau)\right\|\left\|D^{m} v_{\tau}(\tau)\right\| d \tau\right)^{2} \\
\leq & \left.\int_{0}^{t}\left(\left\|D^{m} u(\tau)\right\|^{2}+\left\|D^{m} v(\tau)\right\|^{2}\right)^{\frac{1}{2}}+\left(\left\|D^{m} u_{\tau}(\tau)\right\|^{2}\left\|D^{m} v_{\tau}(\tau)\right\|^{2}\right)^{\frac{1}{2}} d \tau\right)^{2} \\
\leq & \int_{0}^{t}\left(\left\|D^{m} u(\tau)\right\|^{2}+\left\|D^{m} v(\tau)\right\|^{2}\right) d \tau \\
& \int_{0}^{t}\left(\left\|D^{m} u_{\tau}(\tau)\right\|^{2}+\left\|D^{m} v_{\tau}(\tau)\right\|^{2}\right) d \tau .
\end{aligned}
$$

For the last term by using again Cauchy-Schwarz and Young's inequalities we obtain

$$
\begin{aligned}
& 2\left(\left(\left(u, u_{t}\right)+\left(v, v_{t}\right)\right) \int_{0}^{t}\left(D^{m} u(\tau), D^{m} u_{\tau}(\tau)\right)+\left(D^{m} v(\tau), D^{m} v_{\tau}(\tau)\right) d \tau\right) \\
& \leq 2\left(\left(\|u\|^{2}+\|v\|^{2}\right)^{\frac{1}{2}}\left(\left\|u_{t}\right\|^{2}+\left\|v_{t}\right\|^{2}\right)^{\frac{1}{2}}\right) \\
&\left(\int_{0}^{t}\left(\left\|D^{m} u(\tau)\right\|^{2}+\left\|D^{m} v(\tau)\right\|^{2}\right) d \tau \int_{0}^{t}\left(\left\|D^{m} u_{\tau}(\tau)\right\|^{2}+\left\|D^{m} v_{\tau}(\tau)\right\|^{2}\right) d \tau\right)^{\frac{1}{2}} \\
& \leq\left(\left\|u_{t}\right\|^{2}+\left\|v_{t}\right\|^{2}\right) \int_{0}^{t}\left(\left\|D^{m} u(\tau)\right\|^{2}+\left\|D^{m} v(\tau)\right\|^{2}\right) d \tau \\
&(3.15)+\left(\|u\|^{2}+\|v\|^{2}\right) \int_{0}^{t}\left(\left\|D^{m} u_{\tau}(\tau)\right\|^{2}+\left\|D^{m} v_{\tau}(\tau)\right\|^{2}\right) d \tau .
\end{aligned}
$$

Substituting (3.13)-(3.15) into (3.12) becomes

$$
\left(\Phi^{\prime}(t)\right)^{2} \leq 4 \Phi(t)\left(\left(\left\|u_{t}\right\|^{2}+\left\|v_{t}\right\|^{2}\right)+\int_{0}^{t}\left(\left\|D^{m} u_{\tau}(\tau)\right\|^{2}+\left\|D^{m} v_{\tau}(\tau)\right\|^{2}\right) d \tau\right) .
$$


Combining (3.11) and (3.16) we obtain

$$
\begin{aligned}
& \Phi^{\prime \prime}(t) \Phi(t)-\frac{\zeta}{4}\left(\Phi^{\prime}(t)\right)^{2} \\
\geq & \Phi(t)\left(\Phi^{\prime \prime}(t)-\zeta\left(\begin{array}{c}
\left(\left\|u_{t}\right\|^{2}+\left\|v_{t}\right\|^{2}\right) \\
+\int_{0}^{t}\left(\left\|D^{m} u_{\tau}(\tau)\right\|^{2}+\left\|D^{m} v_{\tau}(\tau)\right\|^{2}\right) d \tau
\end{array}\right)\right) \\
\geq & \Phi(t)\left(2\left(\left\|u_{t}\right\|^{2}+\left\|v_{t}\right\|^{2}\right)-2 I(u, v)\right. \\
& \left.-\zeta\left(\left(\left\|u_{t}\right\|^{2}+\left\|v_{t}\right\|^{2}\right)+\int_{0}^{t}\left(\left\|D^{m} u_{\tau}(\tau)\right\|^{2}+\left\|D^{m} v_{\tau}(\tau)\right\|^{2}\right) d \tau\right)\right) .
\end{aligned}
$$

Let

$$
\begin{aligned}
\eta(t)= & (2-\zeta)\left(\left\|u_{t}\right\|^{2}+\left\|v_{t}\right\|^{2}\right)-2 I(u, v) \\
& -\zeta\left(\int_{0}^{t}\left(\left\|D^{m} u_{\tau}(\tau)\right\|^{2}+\left\|D^{m} v_{\tau}(\tau)\right\|^{2}\right) d \tau\right) .
\end{aligned}
$$

By Lemma 2.2 we get

$$
\begin{aligned}
E(0)= & E(t)+\left(\int_{0}^{t}\left\|D^{m} u_{\tau}\right\|^{2} d \tau+\int_{\Omega}\left\|D^{m} v_{\tau}\right\|^{2} d \tau\right) \\
= & \frac{1}{2}\left(\left\|u_{t}\right\|^{2}+\left\|v_{t}\right\|^{2}\right)+\frac{r-2}{2 r}\left(\left\|D^{m} u\right\|^{2}+\left\|D^{m} v\right\|^{2}\right) \\
& +\frac{r-2 \gamma-2}{2 \gamma+2}\left(\left\|D^{m} u\right\|^{2}+\left\|D^{m} v\right\|^{2}\right)^{\gamma+1} \\
& \frac{I(u, v)}{r}+\frac{1}{r^{2}}\left(\|u\|_{r}^{r}+\|v\|_{r}^{r}\right) \\
& +\left(\int_{0}^{t}\left\|D^{m} u_{\tau}\right\|^{2} d \tau+\int_{\Omega}\left\|D^{m} v_{\tau}\right\|^{2} d \tau\right) .
\end{aligned}
$$


Then by combining (3.18) and (3.19), noting $\zeta=\frac{4 C+2 r+4}{C+2}$, which guarantees $2<\zeta<r+2$, and using Lemma 2.1 again, it gives that

$$
\begin{aligned}
& \zeta(t)=(r+2-\zeta)\left(\left\|u_{t}\right\|^{2}+\left\|v_{t}\right\|^{2}\right)-2 r E(0) \\
& +(2 r-\zeta) \int_{0}^{t}\left(\left\|D^{m} u_{\tau}(\tau)\right\|^{2}+\left\|D^{m} v_{\tau}(\tau)\right\|^{2}\right) d \tau \\
& +(r-2)\left(\left\|D^{m} u\right\|^{2}+\left\|D^{m} v\right\|^{2}\right) \\
& +\frac{r-2 \gamma-2}{\gamma+1}\left(\left\|D^{m} u\right\|^{2}+\left\|D^{m} v\right\|^{2}\right)^{\gamma+1} \\
& +\frac{2}{r}\left(\|u\|_{r}^{r}+\|v\|_{r}^{r}\right) \\
& \geq(r+2-\zeta)\left(\left\|u_{t}\right\|^{2}+\left\|v_{t}\right\|^{2}\right)-2 r E(0) \\
& +(r-2)\left(\left\|D^{m} u\right\|^{2}+\left\|D^{m} v\right\|^{2}\right) \\
& \geq(r+2-\zeta)\left(\left\|u_{t}\right\|^{2}+\left\|v_{t}\right\|^{2}\right)-2 r E(0) \\
& +\frac{2(r+2-\zeta)}{C}\left(\left\|D^{m} u\right\|^{2}+\left\|D^{m} v\right\|^{2}\right) \\
& +\left((r-2)-\frac{2(r+2-\zeta)}{C}\right)\left(\left\|D^{m} u\right\|^{2}+\left\|D^{m} v\right\|^{2}\right) \\
& \geq(r+2-\zeta)\left(\left\|u_{t}\right\|^{2}+\left\|v_{t}\right\|^{2}+2\left(\|u\|^{2}+\|v\|^{2}\right)\right)-2 r E(0) \\
& +\left((r-2)-\frac{2(r+2-\zeta)}{C}\right)\left(\left\|D^{m} u\right\|^{2}+\left\|D^{m} v\right\|^{2}\right) \\
& \geq \frac{C(r-2)}{C+2}\left[\left\|u_{t}\right\|^{2}+\left\|v_{t}\right\|^{2}+2\left(\|u\|^{2}+\|v\|^{2}\right)\right. \\
& \left.+\left\|D^{m} u\right\|^{2}+\left\|D^{m} v\right\|^{2}\right]-2 r E(0) \\
& \geq \frac{C(r-2)}{C+2}\left[2\left(u, u_{t}\right)+2\left(v, v_{t}\right)+\left\|D^{m} u\right\|^{2}+\left\|D^{m} v\right\|^{2}\right] \\
& -2 r E(0) \text {. }
\end{aligned}
$$

Therefore by Lemma 3.1 and Lemma 3.2, we conclude that

$$
\begin{aligned}
\zeta(t) & \geq \frac{C(r-2)}{C+2}\left[2\left(u, u_{t}\right)+2\left(v, v_{t}\right)+\left\|D^{m} u\right\|^{2}+\left\|D^{m} v\right\|^{2}\right]-2 r E(0) \\
& =\frac{C(r-2)}{C+2}\left[2\left(u, u_{t}\right)+2\left(v, v_{t}\right)+\left\|D^{m} u\right\|^{2}+\left\|D^{m} v\right\|^{2}-\frac{2 r(C+2)}{C(r-2)}\right] \\
& \geq \frac{C(r-2)}{C+2}\left[2\left(u_{0}, u_{1}\right)+2\left(v_{0}, v_{1}\right)+\left\|D^{m} u_{0}\right\|^{2}+\left\|D^{m} v_{0}\right\|^{2}-\frac{2 r(C+2)}{C(r-2)}\right] \\
& >\sigma_{2}>0,
\end{aligned}
$$

which shows that

$$
\Phi^{\prime \prime}(t) \Phi(t)-\frac{\zeta}{4}\left(\Phi^{\prime}(t)\right)^{2}>\Phi(t) \sigma_{2}>0 .
$$


Let $y(t)=\Phi(t)^{-\frac{\zeta-4}{4}}$, then we obtain

$$
y^{\prime \prime}(t) \leq-\frac{\zeta-4}{4} \sigma_{2} y(t)^{\frac{\zeta}{\zeta-4}}, t \in\left[0, T_{0}\right]
$$

where $\zeta=\frac{4 C+2 r+4}{C+2} \geq 4$.

That is

$$
\lim _{t \rightarrow T^{*}} y(t)=0,
$$

where $T^{*}$ is independent of initial choice of $T_{0}$ and $T^{*}<T_{0}$. Therefore, we can conclude that

$$
\lim _{t \rightarrow T^{*}} \Phi(t)=\infty
$$

\section{Conclusion}

This paper has been able to prove the blow up result for a higher order Kirchhoff type system with logarithmic nonlinearities. This result is new for these types of systems, and it generalises many related problems in the literature.

\section{Acknowledgments}

The authors would like to thank the reviewers and editors of Journal of Universal Mathematics.

\section{Funding}

The author(s) declared that has no received any financial support for the research, authorship or publication of this study.

\section{The Declaration of Conflict of Interest/ Common Interest}

The author(s) declared that no conflict of interest or common interest

\section{The Declaration of Ethics Committee Approval}

This study does not be necessary ethical committee permission or any special permission.

\section{The Declaration of Research and Publication Ethics}

The author(s) declared that they comply with the scientific, ethical, and citation rules of Journal of Universal Mathematics in all processes of the study and that they do not make any falsification on the data collected. Besides, the author(s) declared that Journal of Universal Mathematics and its editorial board have no responsibility for any ethical violations that may be encountered and this study has not been evaluated in any academic publication environment other than Journal of Universal Mathematics.

\section{REFERENCES}

[1] M. M. Al-Gharabli, S. A. Messaoudi, The existence and the asymptotic behavior of a plate equation with frictional damping and a logarithmic source term, Journal of Mathematical Analysis and Applications, 454(2), 1114-1128, (2017). 
[2] Y. Chen, R. Xu, Global well-posedness of solutions for fourth order dispersive wave equation with nonlinear weak damping, linear strong damping and logarithmic nonlinearity, Nonlinear Analysis, 192, 111664, (2020).

[3] S. M. S. Cordeiro, D.C. Pereira, J. Ferreira, C.A Raposo, Global solutions and exponential decay to a Klein-Gordon equation of Kirchhoff-Carrier type with strong damping and nonlinear logarithmic source term. Partial Differential Equations in Applied Mathematics, 3, 100018, (2021).

[4] H. Di, Y. Shang, Z. Song, Initial boundary value problem for a class of strongly damped semilinear wave equations with logarithmic nonlinearity. Nonlinear Analysis: Real World Applications, 51, 102968, (2020).

[5] P. Gorka, Logarithmic Klein-Gordon equation, Acta Physica Polonica B, 40(1), (2009).

[6] X. Han, Global existence of weak solutions for a logarithmic wave equation arising from Q-ball dynamics, Bulletin of the Korean Mathematical Society, 50(1), 275-283, (2013).

[7] T. Hiramatsu, M. Kawasaki, F. Takahashi, Numerical study of Q-ball formation in gravity mediation. Journal of Cosmology and Astroparticle Physics, 2010(06), 008, (2010).

[8] N. Irkıl, E. Pişkin Global existence and decay of solutions for a higher-order Kirchhoff-type systems with logarithmic nonlinearities, Quaestiones Mathematicae, 1-24, (2021), (in press).

[9] G. Kirchhoff, Vorlesungen über Mechanik, 3rd ed., Teubner, Leipzig, 1883.

[10] W. Lian, M. S. Ahmed, R. Xu, Global existence and blow up of solution for semilinear hyperbolic equation with logarithmic nonlinearity, Nonlinear Analysis, 184, 239-257, (2019).

[11] G. Lin, L. Hu, The gloabal attractor for a class of higher-order coupled Kirchhoff-type equations with strong linear damping, European Journal of Mathematics and Computer Science, 4(1), 63-77, (2017).

[12] A. Peyravi, Blow up solutions to a system of higher-order Kirchhoff-type equations with positive initial energy, Taiwanese Journal of Mathematics, 21(4), 767-789, (2017).

[13] E. Pişkin, N.Irkıl, Well-posedness results for a sixth-order logarithmic Boussinesq equation. Filomat, 33(13), 3985-4000, (2019).

[14] E. Pişkin, N.Irkıl, Blow up of the solution for hyperbolic type equation with logarithmic nonlinearity, Aligarh Bulletin of Mathematics, 39 (1-2) ,19-29, (2020).

[15] E. Pişkin, N. Polat, Uniform decay and blow up of solutions for a system of nonlinear higherorder Kirchhoff-type equations with damping and source terms, Contemporary Analysis and Applied Mathematics; 1 (2), 181-199, (2011).

[16] E. Pişkin, E. Harman, Energy Decay of solutions for a system of higher-order Kirchhoff type equations, Journal of New Theory, (29), 89-100, (2019).

[17] X. Wang, Y.Chen, Y. Yang, J. Li, R. Xu, Kirchhoff-type system with linear weak damping and logarithmic nonlinearities, Nonlinear Analysis, 188, 475-499, (2019).

[18] Y. Yang, J. Li, T. Yu, Qualitative analysis of solutions for a class of Kirchhoff equation with linear strong damping term, nonlinear weak damping term and power-type logarithmic source term, Applied Numerical Mathematics, 141, 263-285, (2019).

[19] Q. Hu, H. Zhang, G. Liu, Asymptotic behavior for a class of logarithmic wave equations with linear damping, Applied Mathematics and Optimization, 79(1), 131-144, (2019).

(Nazlı Irkll(Corresponding Author)) Dicle University, Mathematics Department, 21000, DiYARBAKIR, TURKEY

Email address: nazliirkil@gmail.com

(Erhan Pişkin) Dicle University, Mathematics Department, 21000, Diyarbakir, Turkey Email address: episkin@dicle.edu.tr 